\title{
Exercise and nutrition routine improving cancer health (ENRICH): The protocol for a randomized efficacy trial of a nutrition and physical activity program for adult cancer survivors and carers
}

Erica L James ${ }^{1 *}$, Fiona Stacey ${ }^{1}$, Kathy Chapman², David R Lubans ${ }^{3}$, Gabrielle Asprey ${ }^{2}$, Kendra Sundquist ${ }^{2}$, Allison Boyes ${ }^{1,4}$ and Afaf Girgis ${ }^{1,4}$

\begin{abstract}
Background: The Exercise and Nutrition Routine Improving Cancer Health (ENRICH) study is investigating a novel lifestyle intervention aimed at improving the health behaviors of adult cancer survivors and their carers. The main purpose of the study is to determine the efficacy of lifestyle education and skill development delivered via groupbased sessions on the physical activity and dietary behaviors of participants. This article describes the intervention development, study design, and participant recruitment.

Methods/Design: ENRICH is a randomized controlled trial, conducted in Australia, with two arms: an intervention group participating in six, two-hour face-to-face sessions held over eight weeks, and a wait-list control group. Intervention sessions are co-facilitated by an exercise physiologist and dietician. Content includes healthy eating education, and a home-based walking (utilizing a pedometer) and resistance training program (utilizing elastic tubing resistance devices). The program was developed with reference to social cognitive theory and chronic disease self-management models. The study population consists of cancer survivors (post active-treatment) and their carers recruited through community-based advertising and referral from health professionals. The primary outcome is seven-days of sealed pedometry. Secondary outcomes include: self-reported physical activity levels, dietary intake, sedentary behavior, waist circumference, body mass index, quality of life, and perceived social support. The outcomes will be measured at baseline (one week prior to attending the program), eight-weeks (at completion of intervention sessions), and 20-weeks. The intervention group will also be invited to complete 12-month follow-up data collection. Process evaluation data will be obtained from participants by questionnaire and attendance records.
\end{abstract}

Discussion: No trials are yet available that have evaluated the efficacy of group-based lifestyle education and skill development amongst mixed groups of cancer survivors and their carers. The results will have implications for the planning and provision of health and support services during the cancer survivorship phase.

Clinical Trials Registration: Australian New Zealand Clinical Trials Register identifier: ANZCTRN12609001086257.

\footnotetext{
* Correspondence: erica.james@newcastle.edu.au

'School of Medicine and Public Health, University of Newcastle, Hunter

Medical Research Institute, Priority Research Centre for Health Behaviour, Priority Research Centre in Physical Activity and Nutrition, Callaghan NSW

Australia

Full list of author information is available at the end of the article
} 


\section{Background}

The number of cancer survivors worldwide is expected to triple from 25 million in 2008 to 75 million in 2030 [1]. There are approximately 340,000 cancer survivors in Australia, representing about $2 \%$ of the Australian population [2]. Cancer survivors are at increased risk of chronic illnesses such as cardiovascular disease and osteoporosis, death from non-cancer causes, cancer recurrence, secondary cancers, as well as long-term and/ or late effects of treatment, such as fatigue, depression, pain, reduced quality of life (QoL), and weight loss or gain [3-5]. These increased risks can be attributed to cancer treatment, genetic predisposition, and common lifestyle factors [6].

The role of lifestyle factors such as nutrition, physical activity (PA) and a healthy weight aimed at preventing recurrence, secondary cancers, and other chronic diseases is an emerging area of research $[7,8]$. The benefits of PA for people affected by cancer include improved cardiovascular fitness, modest improvements in reducing fatigue, and improved mood and quality of life, body composition, sleep, self-esteem, depression, anxiety, and tiredness $[9,10]$. In breast and bowel cancer survivors, PA is associated with lower risk of disease recurrence and longer survival [11-13]. Resistance training can be safely performed [14], and has been associated with improvements to self esteem, muscular strength, and lean body mass [15]. Evidence is emerging that the pattern of activity is important, with unique metabolic consequences associated with prolonged sedentary behavior [16]. There is a dose-response association between sitting time and mortality from all causes, that is independent of leisure time activity [17], and is associated with the development of bowel, endometrial, ovarian, and prostate cancer, and cancer-specific mortality in women [18].

Diet quality after a breast cancer diagnosis is directly associated with subsequent mental and physical functioning $[19,20]$. Dietary fat reduction and modest weight loss has also been associated with relapse-free survival in post-menopausal breast cancer patients [21]. Both diet and PA contribute to the development of obesity, itself an independent contributor to risk of cancer recurrence and survival $[7,22]$. Evidence suggests that making changes in health behaviors (healthy diet and PA) after a cancer diagnosis may have a significant impact on health [23].

This increasing importance of nutrition and PA for cancer survivors, has been recognized in recent guidelines. An international review by the World Cancer Research Fund and American Institute for Cancer Research concluded that cancer survivors should follow the same diet, healthy weight, and physical activity principles for cancer prevention as the general population [7]. These recommendations are: to be as lean as possible within a healthy body weight; be physically active; limit energy dense food and drink; eat mostly foods of plant origin; limit red meat and avoid processed meat; limit salt; and aim to meet nutritional needs through diet alone [6]. The American College of Sports Medicine and Exercise and Sports Science Australia both acknowledge the safety and efficacy of exercise training for cancer survivors, with general recommendations of low to moderate intensity, three to five times per week, and involving aerobic, resistance, or mixed exercise types [14,24].

Despite these lifestyle recommendations from key agencies and professional organizations, cancer survivors' lifestyle behaviors are similar to the general population. An Australian study of cancer survivors found that unhealthy behaviors (physical inactivity, low fruit and vegetable consumption, overweight/obesity, high alcohol consumption) were similar, if not worse, than a matched sample of persons without a cancer history and survivors were also more likely to report a range of chronic co-morbid conditions [25]. Cancer survivors report being interested in lifestyle behaviors, with some evidence suggesting that a cancer diagnosis may provide a 'teachable moment' to improve health [26].

Little is known about the lifestyle behaviors of the carers of cancer survivors. An Australian study reported that for people with a friend or relative diagnosed with cancer, the diagnosis may have been a cue to make positive diet improvements and increase PA [27]. However, research suggests no difference between carers and non-carers on fruit and vegetable consumption, smoking status, alcohol consumption, PA, healthy body weight, or number or type of chronic illness $[28,29]$. Carers are likely to share many of the same behavioral risk factors as cancer survivors, and would benefit from improvements. Social support appears beneficial in helping cancer survivors make positive changes to exercise behavior [23]. Involving family members in health behavior interventions has positive effects on the patient's adherence to rehabilitation programs, and improved diet and PA behaviors [30,31].

Despite the growing evidence of the benefits and efficacy of lifestyle behaviors in promoting good health and recovery for cancer survivors, there are few services outside of the clinical setting specifically targeting cancer survivors to improve their health. Cancer survivors often report a sense of loss, and feeling "abandoned" or "cast adrift" by the health care system at the time of treatment completion [32]. There is a need to improve support and health services to assist those affected by cancer in the transition from patient to survivor. The main purpose of the present study is to evaluate the 
acceptability and efficacy of a healthy lifestyle (PA and diet) intervention for cancer survivors and their carers.

\section{Methods \\ Study design}

The study utilizes a two-arm randomized controlled trial design with a wait-list control group. Participants complete data collection at baseline, eight and 20 weeks. Intervention participants also complete these measures again at 12 months from baseline. The intervention consists of six, two-hour face-to-face group sessions held over eight weeks. Control group participants are invited to attend ENRICH after completion of the baseline, eight, and 20 week measures. Ethics approval was obtained from the University of Newcastle Human Research Ethics Committee (H-2009-0347). The design, conduct and reporting of this study will adhere to the Consolidated Standards of Reporting Trials (CONSORT) guidelines [33].

\section{Setting}

Three intervention programs and three control programs have been conducted in Sydney, New South Wales, Australia. A further four to six programs are planned to occur in 2011.

\section{Participants}

\section{Selection criteria (eligibility)}

Participation in the program is open to cancer survivors with a previous diagnosis of any type or stage of cancer and who had completed all active treatment (surgery, chemotherapy, radiotherapy, immunotherapy, bone marrow transplants, etc), and who do not have any food restrictions as a result of surgery or treatment, and to carers of cancer survivors. The inclusion criteria of the study are: 1) cancer survivor or carer of cancer survivor; 2) aged 18 years or older; 3) fluent in English; 4) signed medical clearance from their General Practitioner, and 5 ) with a functional performance score of two or less on the Eastern Cooperative Oncology Group criteria (that is "at least ambulatory and capable of all self-care but unable to carry out any work activities or up and about more than 50\% of waking hours") [34]. Both cancer survivors and carers need to meet eligibility criteria. Survivors and carers may participate independently (that is, the survivor does not need a carer to participate and vice versa) or together. A survivor may also bring more than one carer.

\section{Recruitment}

Participants are recruited via multiple methods, including referrals from health professionals, medical centers, professional organizations (such as the Dieticians Association of Australia, New South Wales Oncology Groups), community health centers, cancer support groups, local media, and various Cancer Council NSW resources (website, mailing lists, and publications).

\section{Randomization}

Stratified randomization by age group (less than 50, 50-65, older than 65) and gender is used with a block size of four. A random number sequence for each strata is generated using a random function in SAS version 9.2 and generated a random sequence of A and B's to indicate allocation to intervention or control groups.

The Project Co-ordinator uses a random number table to allocate consenting participants to intervention or control group, stratified by age and gender. If a participant has a partner or carer also consenting, they will be randomized together to the same group, stratified by age and gender of the cancer survivor. The Project Co-ordinator is not blinded to group allocation and participant blinding is not possible due to the wait-list control design of the trial.

\section{Statistical power and sample size}

To detect a mean difference of 2000 steps per day in pedometer-derived step counts between the intervention and control groups with $80 \%$ power and 5\% significance, assuming a standard deviation of 3200 steps, requires 42 subjects per group. The effect size estimate of 2000 steps per day change as the primary outcome is based on published research and clinically meaningful difference [35]. To ensure adequate sample size for secondary outcomes and to account for attrition and missing data, we aim to recruit 75 subjects per group.

\section{Outcomes}

A pen-and-paper survey is completed at baseline (one week prior to first ENRICH program session), eight weeks (at the end of the ENRICH program), 20 weeks from baseline, and for the intervention group only, at 12 months from baseline. At each of these time-points, participants wear a sealed pedometer for seven days and complete a step count diary.

\section{Primary outcome}

The primary outcome is step counts as measured by seven days of sealed pedometry, that is used to provide an average daily step count [36]. Using a sealed pedometer prevents reactivity to monitoring step counts [37]. Despite variation in step counts on different days of the week, three days is the minimum amount of pedometer data required to estimate pedometer-determined PA in a week [38]. The step count diary allows self-report of instances where the pedometer was intentionally removed (eg. swimming, vigorous sports, sleeping) and when the participant forgot to wear the pedometer.

\section{Secondary outcomes}

Physical activity levels PA frequency and duration is measured via eight items from the Active Australia 
survey. The survey evaluates four types of PA in the last week: brisk walking, moderate leisure activity, vigorous leisure activity, and vigorous household or garden chores [39].

Two items assessing the frequency and duration of resistance training were purpose-designed for this study and use the same wording and format of the Active Australia survey questions.

Sedentary behavior Sedentary behavior is measured using a five-item question asking about time spent sitting (hours and minutes) during the last working and non-working day in each of the following domains: (a) while travelling to and from places (e.g., work, shops); (b) while at work; (c) while watching television; (d) while using a computer at home; and (e) at leisure not including watching television (e.g., visiting friends, movies, eating out) [40].

Dietary intake Dietary intake is measured using a food frequency questionnaire, the Dietary Questionnaire for Epidemiological Studies (DQES version 2) [41]. The DQES version 2 contains a list of 74 items across four main categories (1. Cereal foods, sweets \& snacks; 2. Dairy products, meat and fish; 3 . Fruit; 4 . Vegetables) with ten frequency response options ranging from 'Never' to 'three or more times per day'. It also contains three photographs of scaled portions for four foods (used to calculate a portion size calibrator); questions on the overall frequency of consumption of fruits and vegetables (used to calibrate the overestimation of these foods in the food list); and questions on consumption of foods such as bread that do not fit easily into the frequency format. Three questions covering alcohol consumption are also included as part of the DQES [41].

Waist circumference Participants are asked to measure and report their own waist circumference, using standardized instructions [42]. Self-reported waist circumference has been reported as a satisfactory and reliable proxy for objective circumference measures [43].

Body mass index (BMI) Participants report their height and weight, to allow calculation of body mass index. Data from an Australian study reports strong correlations between self-report and clinic measurements, with a trend for self-reported height to be over-estimated (especially in those aged 65 or older), and self-reported weight to be under-estimated [44].

Quality of life Quality of life is assessed using the Medical Outcomes Study Short-Form-12 version 2: a 12-item measure evaluating quality of life across eight health domains: physical functioning (two items), role limitations due to physical health problems (two items), bodily pain (one item), general health perceptions (one item), vitality (energy/fatigue) (one item), social functioning (one item), role limitations because of emotional problems (two items), and general mental health (two items) [45]. These items can be used to calculate two subscales: physical and mental summary scales.

Social support Social support is assessed using the 19item Medical Outcomes Study Social Support Scale and assesses five domains of social support: informational support (four items), affection (three items), tangible support (four items), emotional support (four items), and positive social interaction (four items) [46].

Social cognitive mediators of physical activity Hypothesized social cognitive mediators of PA behavior are assessed using existing validated scales. Due to a lack of validated measures for assessing mediators in relation to diet, these were not included in the survey.

An eight week time reference is provided for all the following social cognitive constructs. "Regular physical activity" is defined as "achieving at least 30 minutes of moderate or vigorous-intensity activity on most, preferably all, days of the week" which is consistent with national guidelines for Australian adults [47].

Behavioral Goal is assessed by asking participants on a scale of 0 per cent to 100 per cent, "How likely is it that you will do regular PA within the next eight weeks?" [48].

Self-Efficacy is measured with a nine-item scale [49]. Participants are asked to rate their confidence $(1=$ not at all confident to $5=$ extremely confident) that they could participate in regular PA over the next eight weeks when: a little tired; in a bad mood or feeling depressed; doing it by themselves; it became boring; there are no noticeable improvements in fitness; having other demands; feeling stiff or sore; there is bad weather; or having to get up early even on weekends.

Outcome Expectations are measured with five items [49]. The items in the scale assessed the extent to which individuals agree or disagree $(1=$ strongly disagree to $5=$ strongly agree) that participating in regular PA over the next eight weeks would for them: reduce tension or manage stress; feel more confident about one's health; sleep better; have a more positive outlook; or help control weight.

Impediments are measured with five items [49]. The items for this scale assess the extent to which individuals agree or disagree $(1=$ strongly disagree to $5=$ strongly agree) that participating in regular PA over the next eight weeks would for them: take too much of my time; have less time for my family and friends; make one too tired because of other daily responsibilities; make one worry about looking awkward if others saw them being physically active; or cost too much money.

Social Support is measured using a two-item scale [48]. Participants are asked whether over the next eight weeks people in their social network are likely to help them participate in regular PA, and whether they feel 
that someone in their social network will provide the support they need in order to be regularly physically active.

Demographics The following demographic information is collected at baseline: gender, year of birth, postcode, marital status, education, current employment, current family income, and smoking status.

Health status At baseline, participants are asked six questions about their health and service use (use of any nutrition and PA support service, diagnosed with chronic health problems, whether they are a cancer survivor, carer, or both, and if a carer, relationship to the cancer survivor), and four questions about cancer diagnosis (type of cancer, when diagnosed, treatments received, status of cancer).

Health Education Impact Questionnaire Cancer survivors are asked to complete the 40-item Health Education Impact Questionnaire (heiQ). The heiQ provides a broad profile of the potential impacts of patient education programs [50] across eight domains: health directed behavior (four items); positive and active engagement in life (five items); emotional well-being (six items); self-monitoring and insight (six items); skill and technique acquisition (four items); constructive attitudes and approaches (five items); social integration and support (five items); and health services navigation (five items) [50].

\section{Process evaluation}

Participant evaluation of the ENRICH program and sessions is measured via a participant program evaluation form (to be completed by participants at the final ENRICH session), and audit of participant attendance lists. The program evaluation form consists of nine questions from the course evaluation module of the heiQ [51]. A further eleven questions evaluating the program were purpose-designed by the research team.

ENRICH program facilitators are asked to provide their feedback on each of the sessions via pen-and-paper form. This enables the facilitators to reflect on any aspects of the program content that did or didn't work; any particular issues not well addressed; and whether or not there were any issues affecting the group sessions.

\section{Procedure}

Participants are asked to complete the pen-and-paper survey, wear their pedometer for seven-days and complete a pen-and-paper step count diary at baseline, eight, and twenty weeks from baseline (see Figure 1). In addition, the intervention group will also complete these measures at 12 months from baseline. Participants who do not return their survey, diary, and pedometer within two weeks receive one reminder call from the Project Co-ordinator. If these materials are not returned after a further two weeks, the Project Co-ordinator phones them to conduct a second and final reminder call.

Participants are asked to attend each of the six ENRICH program sessions. Attendance records are maintained by the Project Co-ordinator.

\section{Intervention development and content}

ENRICH consists of four weekly sessions, followed by two fortnightly sessions. Each session consists of a mix of healthy eating information and activities, a homebased walking program information and resources (with pedometer), and a home-based resistance training program (with elastic tubing equipment, Gymstick ${ }^{\mathrm{TM}}$ ) with information and resources.

ENRICH program sessions are co-facilitated by an exercise physiologist (exercise specialist) and dietician who received training from members of the research team. Educational information is supplemented with practical activities (eg. food label reading), group discussion (eg. brainstorming ideas to overcome barriers to being more active), and role modelling and practice of resistance training exercises, including stretching and appropriate warmups and cool down. The practice is to assist participants to become familiar and comfortable with the equipment, and allows the exercise physiologist an opportunity to ensure correct technique. Participants receive a pedometer, Gymstick $^{\mathrm{TM}}$, step count diary, and written resources as part of the program.

A Gymstick ${ }^{\mathrm{TM}}$ http://www.gymstick.net is a lightweight graphite shaft, with elastic tubing and foot straps that provide resistance and can be used to exercise all of the major muscle groups. It is a safe, effective and relatively inexpensive tool for a strength-based exercise program to be conducted at home [52]. Gymsticks ${ }^{\mathrm{TM}}$ are available in five different resistance levels and the load on each device can be increased by rolling the bar and shortening the elastic tubing [52].

The program content was developed by experts in each of the content areas (members of research team), with input from advisory and working group members, and reviewed by oncology dieticians and physiotherapists. The program content is structured, but allows flexibility in the delivery to allow for discussion, questions, and interactive activities.

The ENRICH program content and delivery approach was guided by Bandura's (2004) Social Cognitive Theory [53] and a chronic disease self-management approach [54]. Examples of social cognitive theory program constructs include: building self-efficacy (self-monitoring of behavior through use of step count diaries); identification of socio-cultural factors such as facilitators and impediments (brainstorming barriers and strategies to exercise when the weather is bad); outcome expectations 


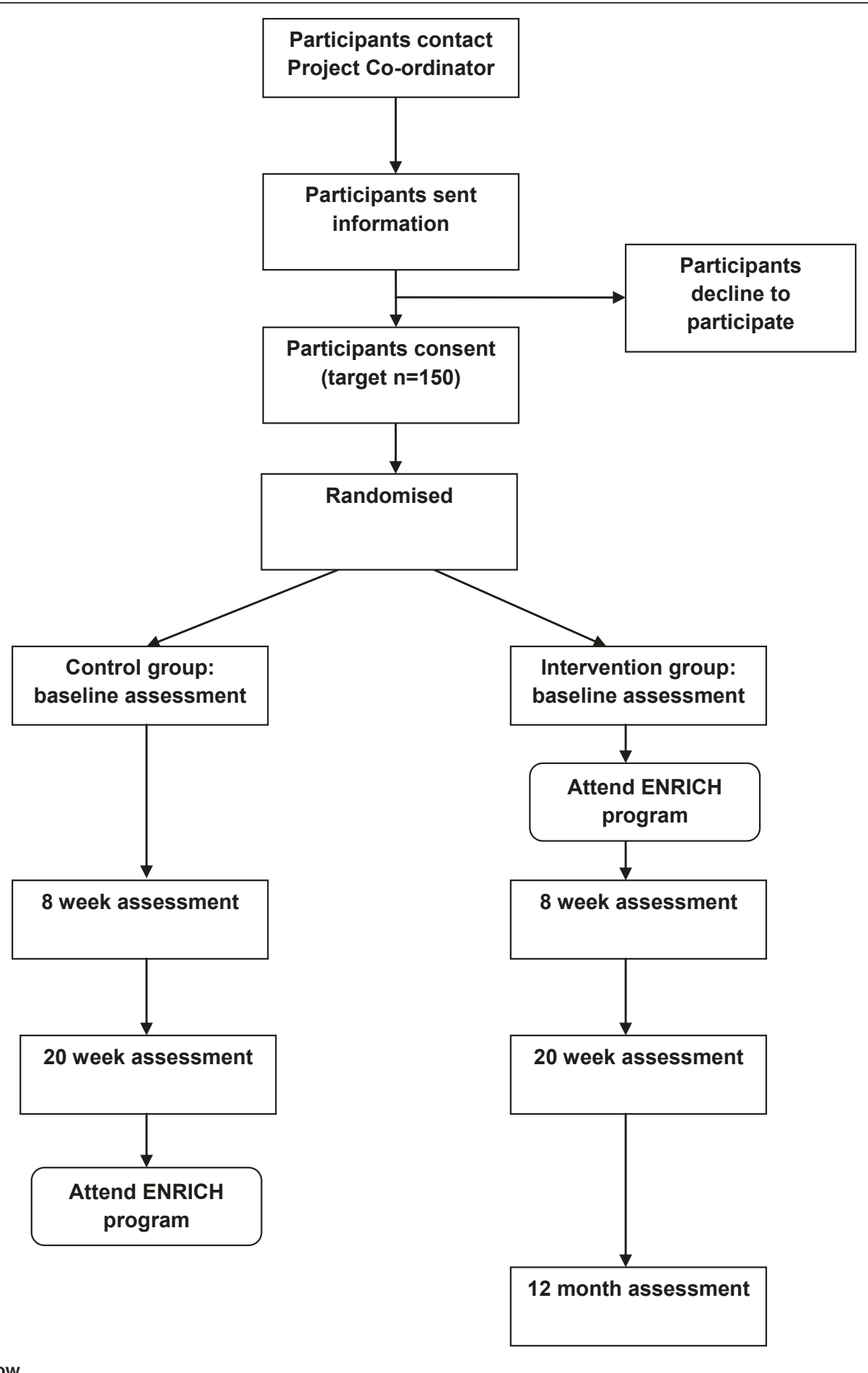

Figure 1 Study flow.

(use and reflection of step count diaries); goal setting (setting and monitoring step count goal); and knowledge of risks and benefits (of PA, healthy eating and healthy weight management).

Traditional chronic disease self-management models encourage participants to take responsibility for their own health and behavior to make sustainable, life-long changes [54]. Chronic disease self-management has similar values to the core social cognitive theory constructs with key elements of chronic disease self-management being self-efficacy, motivation, developing achievable action plans, and role modelling [54]. 


\section{Statistical methods}

Data will be entered into SAS version 9.2. Initial descriptive analyses will be used to describe the sociodemographic and disease characteristics of the participants. Independent t-tests (or other non-parametric equivalents) will be used to assess difference between the intervention and control groups. Paired t-tests (or other non-parametric equivalents) will be used to assess changes in outcomes from baseline to follow-up. Longitudinal data will be analyzed using generalized estimating equation (GEE) models. Analyses will take into account potential clustering of dyad behaviors. Potential mediators of PA behavior change will be assessed using a product-of-coefficients test.

\section{Discussion}

The ENRICH program meets a current gap in the provision of care to cancer survivors and carers, during the survivorship phase. The program focuses on important lifestyle behaviors that have the potential to address long-term and late effects of cancer and treatment, as well as prevention of other chronic health conditions. The program was intentionally developed to be applicable to cancer survivors diagnosed with different cancers and at different stages. The program has the ability to be individually tailored, and because it is not prescriptive, is relevant to all group members. The focus of the program is on giving participants the skills and knowledge to make achievable, life-long change appropriate to the ability of participants.

Limitations of the study include the reliance on selfreport data for body composition and dietary behavior. It was not possible to obtain objective waist and weight measures from the control group participants, without potentially influencing their behaviors during the intervention period.

A novel approach of the program is the emphasis on self-management constructs, and provision of materials for a predominantly home-based PA program. Homebased activity programs place control back with participants who can exercise at a pace and ability at their own comfort level. The use of a home-based resistance training device is also a novel aspect of the program, with the efficacy of an elastic tubing device yet to be demonstrated in this population. As much as ENRICH is designed for participants to implement themselves at home, there is further potential for this program to be modified and tested in different formats that might be applicable to those disadvantaged through distance, isolation, or transport.

\section{Conclusion}

With increasing numbers of cancer survivors at-risk for long-term and/or late effects of treatment and other chronic disease, efforts for promoting the health of this important group are urgently needed. This lifestyle program may provide valuable information relating to the development of other healthy lifestyle interventions for cancer survivors and carers, and result in appropriate behavior change and self-management strategies.

\section{List of abbreviations used}

ENRICH: Exercise and Nutrition Routine Improving Cancer Health; PA: Physical activity; QoL: Quality of life; DQES: Dietary Questionnaire for Epidemiological Studies; BMI: Body mass index; heiQ: Health Education Impact Questionnaire; GEE: Generalized Estimating Equation.

\section{Acknowledgements and funding}

This study was supported by funding from the Australian Better Health Initiative: A joint Australian, State and Territory government initiative with additional funding and infrastructure support provided by the Cancer Council NSW and Hunter Medical Research Institute.

We thank the multi-disciplinary project advisory group (Ms Julie-Anne Mitchell, National Heart Foundation; Ms Katherine Pronk, Liverpool Hospital; Associate Professor Sharon Kilbreath, The University of Sydney; Dr David Dalley, St Vincent's Hospital; Mrs Lisa Oxman, and Ms Anne Norton) who provided input into the overall design and provided valuable advice throughout the project. Thank you to the ENRICH program facilitators: Jennifer Chan, Cate Mellor, Karen Hester, and Belinda Giles, and all of the ENRICH program participants.

The Centre for Health Research and Psycho-oncology (CHeRP) is funded by Cancer Council NSW and University of Newcastle with infrastructure support from the Hunter Medical Research Institute. The views expressed are not necessarily those of the Cancer Council.

\section{Author details}

'School of Medicine and Public Health, University of Newcastle, Hunter Medical Research Institute, Priority Research Centre for Health Behaviour, Priority Research Centre in Physical Activity and Nutrition, Callaghan NSW Australia. ${ }^{2}$ Cancer Council NSW, Woolloomooloo NSW Australia. ${ }^{3}$ School of Education and Priority Research Centre in Physical Activity and Nutrition, University of Newcastle, Callaghan NSW Australia. ${ }^{4}$ Centre for Health Research and Psycho-oncology (CHeRP), University of Newcastle and Cancer Council NSW, Hunter Medical Research Institute, and Priority Research Centre for Health Behaviour, Callaghan NSW Australia.

\section{Authors' contributions}

EJ, KC, and KS conceived the study and obtained the funding. All authors provided input into the study and intervention design. GA and FS were primarily responsible for recruitment and data collection. FS, EJ, KC, and DL were responsible for drafting the manuscript. All authors critically evaluated the article for content and approved the final version.

\section{Competing interests}

The authors declare that they have no competing interests.

Received: 16 March 2011 Accepted: 15 April 2011

Published: 15 April 2011

\section{References}

1. Ferlay J, Shin HR, Bray F, Forman D, Mathers C, Parkin DM: GLOBOCAN 2008, Cancer Incidence and Mortality Worldwide IARC CancerBase No 10. Lyon: IARC; 2010.

2. AlHW (Australian Institute of Health and Welfare), AACR (Australasian Association of Cancer Registries): Cancer in Australia: an overview, 2006 Canberra: AlHW; 2007.

3. Hewitt M, Greenfield S, Stovall E: From cancer patient to cancer survivor. Lost in transition Washington DC: The National Academies Press; 2005.

4. Stanton AL: Psychosocial concerns and interventions for cancer survivors. Journal of Clinical Oncology 2006, 24:5132-5137.

5. Ganz PA: Monitoring the physical health of cancer survivors: a survivorship-focused medical history. Journal of Clinical Oncology 2006, 24:5105-5111. 
6. Demark-Wahnefried W, Pinto BM, Gritz ER: Promoting health and physical function among cancer survivors: potential for prevention and questions that remain. Journal of Clinical Oncology 2006, 24:5125-5131.

7. World Cancer Research Fund/American Institute for Cancer Research: Food, Nutrition, Physical Activity, and the Prevention of Cancer: a Global Perspective Washington DC: AICR; 2007.

8. Doyle C, Kushi LH, Byers T, Courneya KS, Demark-Wahnefried W, Grant B, McTiernan A, Rock CL, Thompson C, Gansler T, et al: Nutrition and physical activity during and after cancer treatment: an American Cancer Society guide for informed choices. CA A Cancer Journal for Clinicians 2006, 56:323-353.

9. Irwin ML: Physical activity interventions for cancer survivors. British Journal of Sports Medicine 2009, 43:32-38.

10. Kirshbaum MN: A review of the benefits of whole body exercise during and after treatment for breast cancer. Journal of Clinical Nursing 2007, 16:104-121.

11. Speed-Andrews AE, Courneya KS: Effects of exercise on quality of life and prognosis in cancer survivors. Current Sports Medicine Reports 2009, 8:176-181

12. Irwin ML, Mayne ST: Impact of nutrition and exercise on cancer survival. The Cancer Journal 2008, 14:435-441.

13. Vrieling A, Kampman E: The role of body mass index, physical activity, and diet in colorectal cancer recurrence and survival: a review of the literature. American Journal of Clinical Nutrition 2010, 92:471-490.

14. Schmitz KH, Courneya KS, Matthews C, Demark-Wahnefried W, Galvao DA, Pinto BM, Irwin ML, Wolin KY, Segal RJ, Lucia A, et al: American College of Sports Medicine roundtable on exercise guidelines for cancer survivors. Medicine \& Science in Sports and Exercise 2010, 42:1409-1426.

15. Courneya KS, Segal RJ, Mackey JR, Gelmon K, Reid RD, Friedenreich CM, Ladha AB, ProulX C, Vallance JKH, Lane K, et al: Effects of aerobic and resistance exercise in breast cancer patients receiving adjuvant chemotherapy: a multicenter randomized controlled trial. Journal of Clinical Oncology 2007, 25:4396-4404.

16. Lynch BM, Dunstan DW, Healy GN, Winkler E, Eakin E, Owen N: Objectively measured physical activity and sedentary time of breast cancer survivors, and associations with adiposity: findings from NHANES (20032006). Cancer Causes \& Control 2010, 21:283-288.

17. Katzmarzyk PT, Church TS, Craig CL, Bouchard C: Sitting time and mortality from all causes, cardiovascular disease, and cancer. Medicine \& Science in Sports and Exercise 2009, 41:998-1005.

18. Lynch BM: Sedentary behavior and cancer: a systematic review of the literature and proposed biological mechanisms. Cancer Epidemiology, Biomarkers \& Prevention 2010, 19:2691-2709.

19. Wayne SJ, Baumgartner K, Baumgartner RN, Bernstein L, Bowen DJ, BallardBarbash R: Diet quality is directly associated with quality of life in breast cancer survivors. Breast Cancer Research and Treatment 2006, 96:227-232.

20. Demark-Wahnefried W, Clipp EC, Morey MC, Pieper CF, Sloane R, Snyder DC, Cohen HJ: Physical function and associations with diet and exercise: Results of a cross-sectional survey among elders with breast or prostate cancer. International Journal of Behavioral Nutrition and Physical Activity 2004, 1.

21. Blackburn GL, Wang KA: Dietary fat reduction and breast cancer outcome: results from the Women's Intervention Nutrition Study (WINS). American Journal of Clinical Nutrition 2007, 86(suppl):8785-881S.

22. Toles M, Demark-Wahnefried W: Nutrition and the cancer survivor: evidence to guide oncology practice. Seminars in Oncology Nursing 2008, 24:171-179.

23. Park CL, Gaffey AE: Relationships between psychosocial factors and health behavior change in cancer survivors: an integrative review. Annals of Behavioral Medicine 2007, 34:115-134.

24. Hayes SC, Spence RR, Galvão DA, Newton RU: Australian Association for Exercise and Sport Science position stand: optimising cancer outcomes through exercise. Journal of Science and Medicine in Sport 2009, 12:428-434.

25. Eakin EG, Youlden DR, Baade PD, Lawler SP, Reeves MM, Heyworth JS, Fritschi L: Health behaviors of cancer survivors: data from an Australian population-based survey. Cancer Causes \& Control 2007, 18:881-894.

26. Demark-Wahnefried W, Aziz NM, Rowland JH, Pinto BM: Riding the crest of the teachable moment: promoting long-term health after the diagnosis of cancer. Journal of Clinical Oncology 2005, 23:5814-5830.
27. Humpel N, Magee C, Jones SC: The impact of a cancer diagnosis on the health behaviors of cancer survivors and their family and friends. Supportive Care in Cancer 2007, 15:621-630.

28. McGuire L, Bouldin EL, Andresen EM, Anderson LA: Examining modifiable health behaviors, body weight, and use of preventive health services among caregivers and non-caregivers aged 65 years and older in Hawaii, Kansas, and Washington using 2007 BRFSS. The Journal of Nutrition, Health \& Aging 2010, 14:373-379.

29. Son KY, Park SM, Lee CH, Choi GJ, Lee DG, Jo SH, Lee SH, Cho BL: Behavioral risk factors and use of preventive screening services among spousal caregivers of cancer patients. Supportive Care in Cancer 2010.

30. Martire LM, Lustig AP, Schulz R, Miller GE, Helgeson VS: Is it beneficial to involve a family member? A meta-analysis of psychosocial interventions for chronic illness. Health Psychology 2004, 23:599-611.

31. Daly J, Sindone AP, Thompson DR, Hancock K, Chang E, Davidson P: Barriers to participation in and adherence to cardiac rehabilitation programs: a critical literature review. Progress in Cardiovascular Nursing 2002, 17:8-17.

32. Jefford M, Karahalios E, Pollard A, Baravelli C, Carey M, Franklin J, Aranda S, Schofield P: Survivorship issues following treatment completion - results from focus groups with Australian cancer survivors and health professionals. Journal of Cancer Survivorship 2008, 2:20-32.

33. Moher D, Hopewell S, Schulz KF, Montori V, Gotzsche PC, Devereaux PJ, Elbourne D, Egger M, Altman DG: CONSORT 2010 explanation and elaboration: updated guidelines for reporting parallel group randomised trials. British Medical Journal 2010, 340:c869.

34. Oken MM, Creech RH, Tormey DC, Horton J, Davis TE, McFadden ET, Carbone PP: Toxicity and response criteria of the Eastern Cooperative Oncology Group. American Journal of Clinical Oncology 1982, 5:649-655.

35. Bravata DM, Smith-Spangler C, Sundaram V, Gienger AL, Lin N, Lewis R, Stave CD, Olkin I, Sirard JR: Using pedometers to increase physical activity and improve health. A systematic review. Journal of the American Medical Association 2007, 298:2296-2304.

36. Tudor-Locke C, Myers AM: Methodological considerations for researchers and practitioners using pedometers to measure physical (ambulatory) activity. Research Quarterly for Exercise and Sport 2001, 72:1-12.

37. Marshall AL: Should all steps count when using a pedometer as a measure of physical activity in older adults? Journal of Physical Activity and Health 2007, 4:304-314

38. Tudor-Locke C, Burkett L, Reis JP, Ainsworth BE, Macera CA, Wilson DK: How many days of pedometer monitoring predict weekly physical activity in adults? Preventive Medicine 2005, 40:293-298.

39. Australian Institute of Health and Welfare (AlHW): The Active Australia Survey: a guide and manual for implementation, analysis and reporting Canberra: AlHW; 2003.

40. Marshall AL, Miller YD, Burton NW, Brown WJ: Measuring total and domain-specific sitting: a study of reliability and validity. Medicine \& Science in Sports and Exercise 2010, 42:1094-1102.

41. Cancer Epidemiology Centre Nutritional Assessment Office: Dietary Questionnaire for Epidemiological Studies (DQES v2) User Information Guide 2009 Carlton: Cancer Council Victoria; 2009.

42. Data Dictionary Supplement of the ALSWH (The Australian Longitudinal Study on Women's Health). [http://www.alswh.org.au/InfoData/dictsupp. htmll].

43. Rimm EB, Stampfer MJ, Colditz GA, Chute CG, Litin LB, Willett WC: Validity of self-reported waist and hip circumferences in men and women. Epidemiology 1990, 1:466-473.

44. Taylor AW, Dal Grande E, Gill TK, Chittleborough CR, Wilson DH, Adams RJ, Grant JF, Phillips P, Appleton S, Ruffin RE: How valid are self-reported height and weight? A comparison between CATI self-report and clinic measurements using a large cohort study. Australian and New Zealand Journal of Public Health 2006, 30:238-246.

45. Ware JE Jr, Kosinski M, Keller S: A 12-item short-form health survey: Construction of scales and preliminary tests of reliability and validity. Medical Care 1996, 34:220-233.

46. Sherbourne CD, Stewart AL: The MOS social support survey. Social Science \& Medicine 1991, 32:705-714.

47. Department of Health and Ageing: National Physical Activity Guidelines for Australians Canberra; 1999. 
48. Courneya KS, Plotnikoff RC, Hotz SB, Birkett NJ: Social support and the theory of planned behavior in the exercise domain. American Journal of Health Behavior 2000, 24(4):300.

49. Plotnikoff RC, Blanchard C, Hotz SB, Rhodes R: Validation of the decisional balance scales in the exercise domain from the transtheoretical model: a longitudinal test. Measurement in Physical Education and Exercise Science 2001, 5:191-206.

50. Osborne RH, Elsworth GR, Whitfield K: The Health Education Impact Questionnaire (heiQ): An outcomes and evaluation measure for patient education and self-management interventions for people with chronic conditions. Patient Education and Counseling 2007, 66:192-201.

51. heiQ Items by Domain V3.0. [http://www.crd.unimelb.edu.au/heiq/].

52. Lubans DR, Aguiar EJ, Callister R: The effects of free weights and elastic tubing resistance training on physical self-perception in adolescents. Psychology of Sport and Exercise 2010, 11:497-504.

53. Bandura A: Health promotion by social cognitive means. Health Education \& Behavior 2004, 31:143-164.

54. Lorig K: Chronic disease self-management. The American Behavioral Scientist 1996, 39:676-683.

\section{Pre-publication history}

The pre-publication history for this paper can be accessed here: http://www.biomedcentral.com/1471-2458/11/236/prepub

doi:10.1186/1471-2458-11-236

Cite this article as: James et al:: Exercise and nutrition routine improving cancer health (ENRICH): The protocol for a randomized efficacy trial of a nutrition and physical activity program for adult cancer survivors and carers. BMC Public Health 2011 11:236.

\section{Submit your next manuscript to BioMed Central and take full advantage of:}

- Convenient online submission

- Thorough peer review

- No space constraints or color figure charges

- Immediate publication on acceptance

- Inclusion in PubMed, CAS, Scopus and Google Scholar

- Research which is freely available for redistribution

Submit your manuscript at www.biomedcentral.com/submit 